\title{
Vulnerability and risk perception in the management of HIVIAIDS: Public priorities in a global pandemic
}

\author{
Peter Tsasis ${ }^{1,2}$ \\ N. Nirupama ${ }^{2}$ \\ 'School of Health Policy \\ and Management, ${ }^{2}$ School of \\ Administrative Studies, York \\ University, Toronto, Ontario, Canada
}

\begin{abstract}
Understanding the way perception of risk is shaped and constructed is crucial in understanding why it has been so difficult to mitigate the spread of HIV/AIDS. This paper uses the Pressure and Release (PAR) model, used to predict the onset of natural disasters as the conceptual framework. It substitutes vulnerability and risk perception as the trigger factors in the model, in making the case that HIV/AIDS can be characterized as a slow onset disaster. The implications are that vulnerability must be managed and reduced by addressing root causes, dynamic pressures, and unsafe conditions that contribute to the HIV/AIDS pandemic. HIV/ AIDS programs must be culturally appropriate and work toward influencing risk perception, while addressing social norms and values that negatively impact vulnerable populations. By impacting cultural and social expectations, individuals will be able to more readily adopt safer sex behaviors. The development of policies and programs addressing the issues in context, as opposed to individual behaviors alone, allows for effective public health intervention. This may have implications for public health measures implemented for combating the spread of HIV/AIDS.
\end{abstract}

Keywords: vulnerability, risk perception, HIV/AIDS, public health intervention

\section{Introduction}

Tsunamis, earthquakes, and other natural disasters throughout the history of civilization have captured worldwide attention, demanding an immediate response. Increasingly with sophisticated technology, a global response to disasters has been comprehensive with immediate aid and prevention plans made against further catastrophe. Yet, the human immunodeficiency virus (HIV), which causes the slow deterioration of the immune system leading to an acute immune deficiency syndrome (AIDS) has created chaos within social structures, devastated communities, and killed millions without receiving the same swift response as natural disasters. According to the Joint United Nations Programme on HIV/AIDS (UNAIDS), approximately 33.2 million people are currently inflicted with HIV, which makes HIV/AIDS a pandemic. ${ }^{1}$ Even though HIV/AIDS is viewed as a pandemic with potential for catastrophe, many populations around the world continue to neglect the severe risk involved in practices that make them vulnerable to HIV/AIDS. Since risk perception is embedded and impacted by the various cultures of the world, it is not surprising that the spread of HIV/AIDS is so varied in many regions of the world. Perhaps, the issue lies in understanding risk and how it interplays with HIV/AIDS.

A wide range of risk theories developed over the past decade have incorporated the influence of varying ideologies in explaining the way we perceive risk. ${ }^{2-5}$ Understanding the way perception of risk is shaped and constructed is crucial in understanding why it has been so difficult to mitigate the spread of HIV/AIDS. The association between HIV infection and the perception of risk in different regions of the world has emphasized 
the need to reevaluate the public health measures being implemented to control the spread of HIV/AIDS, particularly for those people most at risk. This paper looks at HIV/AIDS and the devastating effects the pandemic is having on populations with diverse risk perceptions of the disease, and makes the case that HIV/AIDS can be characterized as a slow onset disaster.

\section{Risk perception and HIVIAIDS}

Risk perception varies in that risk perception is linked to an individual's predisposition to be risk-averse or risk-seeking and to the individual's knowledge regarding the object or situation at hand. ${ }^{2}$ However, the unpredictability of hazards and uneven distribution of knowledge and access to knowledge in societies means that members of the public are not always in a position to define and understand risk. At some point, individuals may lack the ability and opportunity to decide which risks affect them and to what extent. Often the public is forced to place their trust in social structures that are viewed as acting in their best interests. ${ }^{3}$

Since different groups and stakeholders have different interests at the level of public debate, certain dangers are attached to particular threats when different perceptions of risk are created. ${ }^{4}$ Both social institutions and social structures thus harbor the power to shape risk perception. ${ }^{4}$ This process of negotiating risk demonstrates how people organize their universe through cultural and social biases and choose what to fear based on their way of life and patterns of cultural and social norms. ${ }^{2}$ These biases cause selective attention to risk and preferences for different types of risk taking behaviors, informed by an inherent compulsion to defend one's way of life. ${ }^{2}$

Furthermore, although it is ultimately social structures that define and shape risk perception in societies, we see that risk is usually individualized, leading to worry and anxiety among persons regarding specific threats that have yet to take place..$^{5}$ Through this process of individualization, risk becomes associated with choice, responsibility, and blame, and the individual rather than society is held accountable for negative outcomes. ${ }^{6}$

One of the peculiarities of risk is that the knowledge of risk is not in-sync with the actions that should be taken. ${ }^{4}$ In other words, the principle of taking the greatest precaution for the worst possible outcome is not executed. Although it is possible that this is due to lack of awareness, the more likely explanation is the lack of acceptance. Research regarding risk perception demonstrates that risk that is (1) involuntary, (2) unfamiliar, and (3) potentially catastrophic is the most difficult for people to accept. ${ }^{5}$ Acquiring HIV/AIDS is an involuntary occurrence for most. Lack of knowledge, but more often, lack of control over social and economic circumstances precipitates individuals to engage in risky behavior that leads to the transmission of HIV/AIDS. In many cultures, for example, women have little power over their sexuality and the sexual practices in which they engage. ${ }^{7}$ In addition, poverty can lead to both male and female prostitution. In these cases, individuals place themselves at high risk for acquiring HIV/AIDS in trying to avoid social exclusion, violence, and poverty. ${ }^{7}$

The risk of contracting HIV/AIDS may also be unfamiliar to many. The perception that HIV/AIDS occurs only amongst homosexuals is still prevalent. ${ }^{8,9}$ In addition, because the symptoms of AIDS do not take full effect for as many as 8 to 10 years from the time of infection, many are unaware of being seropositive, and those who do know may not fully comprehend or accept the magnitude of the disease.

Finally, HIV/AIDS is catastrophic. The numbers speak for themselves; according to the 2007 UNAIDS Global AIDS Epidemic Update, an estimated 33.2 million living with HIV, 2.5 million newly infected, and 2.1 million individuals lost lives due to HIV/AIDS world-wide at the end of $2006 .{ }^{1}$ However, because of the stigma attached to being HIV-positive in many communities, it is likely that cases of HIV/AIDS are underreported and thus these numbers may actually be considerably higher. ${ }^{10}$

Although risk perception may be clouded by the individual's inability to accept the reality of risks that are involuntary, unfamiliar, and catastrophic, the problem is not necessarily with the individual, but rather with society at large. Within the discourse of public health, health risks have been individualized such that it is an individual's choice to engage in certain behaviors that cause the individual to acquire HIV/AIDS. ${ }^{5}$ This view has led to the labeling of particular groups of individuals and populations as "at-risk". 5 Populations deemed at-risk for HIV/AIDS include sex workers, men who have sex with men (MSM), and injection drug users. This narrow definition of those at-risk can also be misleading considering that heterosexual and mother-to-child transmission of HIV/AIDS is increasing rapidly across populations. ${ }^{11}$ As a result this has led to a limited focus on awareness and education as solutions, and has allowed those in power to dehumanize, blame, and avoid responsibility for those suffering from HIV/AIDS. ${ }^{12}$

\section{Modeling the HIVIAIDS disaster}

Disasters are often referred to as unplanned, socially disruptive events with extreme effects. ${ }^{13}$ The characteristics 
common to environmental and natural disasters mirror the ways in which HIV/AIDS destroys and impacts communities. These characteristics include a high impact on individuals or populations; the spanning of spatial and temporal boundaries; large-scale damage to human life; and root causes that are complex. ${ }^{13}$ With regards to the latter, disasters are triggered not by a single event, but rather through the interaction of a multitude of factors and a buildup of unnoticed events. This is particularly true for HIV/AIDS.

The vulnerability of a population to HIV/AIDS is rooted in social processes and underlying causes that may actually be quite unrelated to the end result itself, namely the contraction of HIV/AIDS. ${ }^{14}$ The factors that create vulnerability in populations can be modeled to provide a visual representation of the potential negative impact of HIV/AIDS. Since risk and risk perception are a function of the degree of vulnerability and the hazard type, the Pressure and Release (PAR) model first developed by Wisner and colleagues can be used and adapted to depict the root causes and process of HIV/AIDS transmission. The PAR model is generally used to outline how disasters are shaped by external conditions that apply increasing pressure until a release is forced resulting in a disaster. ${ }^{14}$ This build-up of pressure is referred to as a "progression of vulnerability" that consists of three stages: (1) root causes, (2) dynamic pressure, and (3) unsafe conditions. ${ }^{14}$

By building on the PAR model, we can identify sources of vulnerability as root causes of HIV/AIDS transmission which center around political instability, poverty, and unequal access to power and resources. ${ }^{14}$ Countries such as South Africa, Zimbabwe, and Kenya have some of the highest HIV/AIDS infection rates since they are nations with populations experiencing unequal access or lack of resources, poverty, social inequality, and instability. ${ }^{1}$

In the second stage of the model, vulnerability increases via dynamic processes that reduce the ability of the population or risk group to handle adverse circumstances. Here, local markets and fluctuating systems of labor play a major contributory role to a disaster. ${ }^{14}$ Finally, vulnerability peaks due to unsafe conditions where the physical and social environment of the population is unsanitary and/or hostile. As vulnerability increases, so does the risk to the population.

Figure 1 illustrates the HIV/AIDS pandemic in the context of the PAR model. The progression of vulnerability, paired with the hazard of HIV/AIDS creates the ideal setting for rapid HIV/AIDS transmission (the risk). In other words, risk equates to vulnerability (V) multiplied by the level of hazard $(\mathrm{H})$ that exists. In this context, risk is defined as the probability that a person may acquire the HIV infection.
Vulnerability to risk is shaped by three factors. First is the resilience of a population, or the capacity of the people to resist and recover from the outcomes of a disaster. ${ }^{15}$ The second component is the health of the population, or the robustness of individuals, which is most influenced by 'livelihood' and the availability of social operations such as healthcare services. ${ }^{15}$ The final factor determining vulnerability is the degree of preparedness of a population. The level of preparedness is shaped by societal values and beliefs, which determine what is viewed as a risk and in turn which measures are taken for protection, if any. ${ }^{15}$ It is interesting to note that in Figure 1, factors such as poverty and inadequate government assistance act not only as root causes, but also as dynamic pressures and unsafe conditions. Needless to say, HIV/AIDS is most prevalent in populations where social inequality persists and where the disempowered are victimized. ${ }^{16}$

\section{HIVIAIDS as a global concern}

Although a stabilization of the HIV/AIDS epidemic has been noted in the last few years, the infection continues to spread rapidly in actual numbers. ${ }^{17}$ Of the many reasons why HIV/AIDS stands out as atypically disastrous is that its impact is gradual and thus referred to as slow onset. ${ }^{18}$ Although disasters are often thought of as sudden, the worst consequences of disasters are not necessarily felt at the point of occurrence and can easily emerge long after the causes and effects have been identified. ${ }^{13}$ HIV/AIDS is one such disaster. The inability of populations and in some cases complete disregard of governments to recognize risk factors, symptoms, and infection rates prevents appropriate response measures from being implemented. ${ }^{18}$ It is unfortunate that by the time a response is mounted by public authorities; numerous individuals have already been infected who may not have been infected if early prevention measures were in place.

Nowhere is the epidemic more pronounced than in sub-Saharan Africa where over the past decade the epidemic crossed the continent through migrants and refugees. ${ }^{14}$ With just over $10 \%$ of the world's population living in this region, it is disturbing that almost $64 \%$ are living with HIV/AIDS. ${ }^{1}$ Despite the concentration of HIV/AIDS cases in Africa, the disease continues to spread in other parts of the world as well. Since the breakup of the Union of Soviet Socialist Republics (USSR), the resulting fifteen new nations have been experiencing great political, social and economic instability. ${ }^{19}$ This social and political instability has led to increased poverty, driving many to resort to substance abuse. Subsequently, injection drug use and commercial sex work are both dominant transmission paths for HIV/AIDS in this region. ${ }^{17}$ 


\section{Root causes}

\begin{tabular}{|l|}
\hline - Limited Access to: \\
-resources for stable \\
livelihoods \\
-land \& stock for labor \& \\
agricultural production \\
-power due to situated \\
hierarchical structures \\
-comprehensive health care \\
services \\
- Lack of: \\
-adequate governmental \\
intervention to prioritize HIV \\
on political agenda \\
-aid from international/foreign \\
relations \\
-screening for HIV-infected \\
population including mothers of \\
unborn children \\
• Poverty \\
- New Epidemics Rising \\
Untouched Regions \\
- Inequality \\
-Gender inequality \\
-Disempowered women \\
- Unaware HIV-positive \\
pregnant mothers \\
-Specific economic \\
systems
\end{tabular}

Dynamic pressures

- Constant concerns re:

-variation in strain of HIV

(formation of recombinants)

-variability of spread by region and access to ARVs

-pathogenic activity of HIV

causing delayed, dormant, and

hidden symptoms

- Population growth

-Majority in underdeveloped nations; sustained poverty

- Migration in and out of infected regions

- Lack of:

-local production in markets

-healthy family members for support in laborious work

-access to contraceptives and

knowledge re: sexual

intercourse, STDs, \& AIDS

- Governmental problems re:

-inability of impoverished states

to repay wealthier ones

-corruption and hidden political

ploys at different levels

of government

-war and conflict leading

to displaced citizens

- decreased/limited access to food

due to shortage during

famine/drought

- Re-emerging risky behavio

- Globalization causing mixing

of HIV-infected (not limited to

neighboring cities

or countries)

\section{Unsafe conditions}

HAZARD

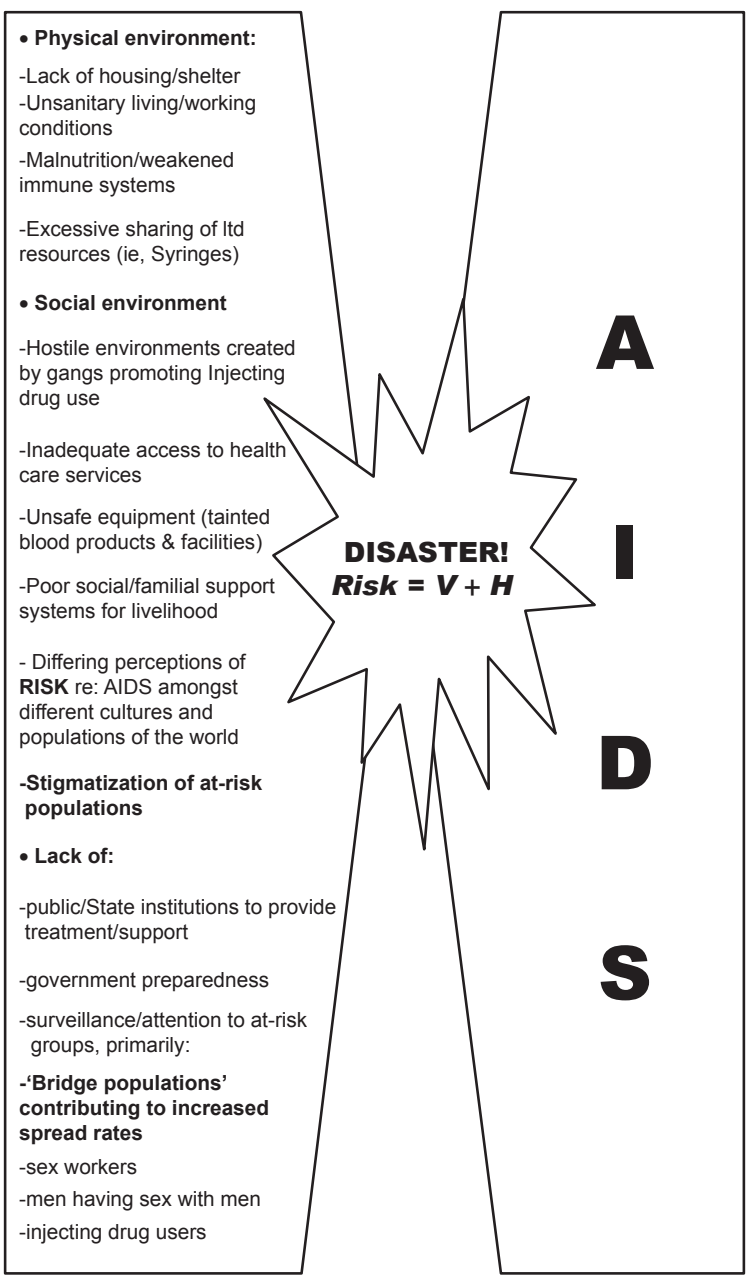

Figure I The progression of vulnerability to AIDS within the context of the PAR model.

The Russian Federation and Ukraine alone make up 1.3 million of the 1.5 million cases in Eastern Europe. ${ }^{17}$

In Asia, an estimated 4.9 million people were living with HIV/AIDS in 2007, with significant growth in prevalence in Indonesia and Vietnam. ${ }^{1}$ Injection drug use is a primary transmission method in this area. In Jakarta, Indonesia $40 \%$ of injection drug users tested positive for HIV in $2005 .{ }^{1}$ In Vietnam, HIV prevalence among injection drug users increased from 9\% in 1996 to 34\% in 2005. ${ }^{1}$ Furthermore, in most Asian countries condom use remains very low. This cultural norm is contributing to increasing infection rates among male and female sex workers. Like their international counterparts, the HIV/AIDS epidemic in Asia is an outcome of social forces, including population mobility, environmental degradation, economic upheaval, and poverty.

HIV/AIDS cases in Latin America are also on the rise. Much of this increase is attributed to the street culture present in many Latin countries that encourages illicit drug use and unsafe sex, particularly among young males. Young boys in Brazil, for example, are encouraged to lose their virginity to demonstrate their masculinity. ${ }^{20}$ In Latin American nations, HIV prevalence is on the rise with populations at risk. The Latino population outside of Latin America also has an increased risk of acquiring HIV/AIDS due to 
cultural factors that contribute to sustained participation in risky behavior. ${ }^{21}$

Despite the broad reach of HIV/AIDS across the globe, governments have failed to acknowledge that HIV/AIDS remains an ongoing crisis and populations continue to neglect the severe risk involved in practices that make them vulnerable to HIV/AIDS. ${ }^{12}$ The most vulnerable population groups for HIV/AIDS are women and children. There are approximately 2.5 million children across the world that are living with HIV/AIDS. ${ }^{1}$ In 2006 alone, an estimated 420,000 new AIDS cases arose in children under the age of 15 years and the prevalence rate in pregnant women has also increased significantly worldwide. ${ }^{1}$ Furthermore, more than $40 \%$ of new infections worldwide are from the young population in the range of 15-24 years of age, indicating a sustenance or prolonging of the virus in populations of the generations to come. ${ }^{1}$ Thus, understanding the way perception of risk is shaped and constructed is crucial in identifying why it has been so difficult to mitigate the spread of HIV/AIDS.

\section{Discussion}

\section{The role of public health}

The spread of HIV/AIDS is exacerbated by social factors that include not only socio-economic status, political instability, and geographic location, but also gender and sexual practice. ${ }^{14}$ Perceptions of risk in contracting HIV/AIDS are shaped by related patterns of social relations and cultural biases. The power to shape risk perception is usually in the hands of very few; namely those with control over social, political, and/or economic institutions.

The case can be made that HIV/AIDS causes widespread loss and serious disruption to the functioning of a community, much like an environmental disaster. ${ }^{23}$ Yet, despite the potential of HIV/AIDS to become a global disaster, many populations continue to neglect the risk involved in practices that make them vulnerable to HIV/AIDS. This failure to mitigate the spread of the infection may be due to the fact that perceptions of risk are shaped by patterns of social relations and cultural biases. In addition, the power to shape risk perception is usually limited to those with control over social, political, and/or economic institutions. Since the social, political, and economic context plays a role in HIV/ AIDS-related risk perception, there is a need to reevaluate public health measures being implemented to control the spread of HIV/AIDS.

The purpose of public health is to manage threats to the health of a population through preventative measures and treatment. Although HIV/AIDS is not only a health problem, but also a developmental issue, attempts at mitigating the spread of the infection typically occur solely through the health sector.

Risk perception is culturally influenced and therefore risky behavior is a social rather than an individual issue. However, the mass media often perpetuates risk as an individual issue and unique to selected populations (intravenous drug users, commercial sex workers and men who have sex with men [MSM]). Therefore, the role of media must also be recognized in terms of being an important player in disseminating information on risks as well as promoting a cultural approach to prevent further spread of HIV/AIDS among a population where social instability exists. As a result, the media plays an integral role in shaping or reinforcing risk behavior as an individual phenomenon, where the individual chooses to be in that risk group. ${ }^{24,25}$ Public health measures implemented to combat the spread of HIV/AIDS must be re-evaluated with consideration for ways in which culture and media shape risk perception, particularly for those groups most at-risk. In using the PAR model as a guide, vulnerability must be managed and reduced by addressing root causes, dynamic pressures, and unsafe conditions that contribute to the HIV/AIDS pandemic. HIV/AIDS programs must be culturally appropriate and work toward influencing risk perception, while addressing social norms and values that negatively impact vulnerable populations such as women and children.

Prevention programs typically focus on raising awareness and using interpersonal strategies, such as peer education and testimonials to influence behavior change. These initiatives have had mixed results because often those most at risk are those in poverty, and these individuals have limited means for effecting change in their circumstances. ${ }^{26}$ For this reason, prevention programs aimed at individual behavior change can be ineffective in many contexts. ${ }^{27}$ Even awareness campaigns that are deemed "culturally-appropriate" have produced mixed results with regards to effectiveness. ${ }^{26}$ These approaches to managing HIV/AIDS fail to consider the evidence that risk perception is culturally influenced and therefore risky behavior is a social rather than an individual issue. It is wrong to assume that decision-making and behavior are always rational. Social and economic factors such as relationships, community expectations, and access to resources have a major impact on behavior, and at times may prevent individuals from adopting safe sex practices that prevent HIV/AIDS transmission. This indicates that attempting to reform behavior to reduce HIV transmission risk is unlikely without structural changes - that is, public health interventions aimed at changing the environment 
rather than individual behavior may be more successful in reducing the spread of the infection.

Public health measures to combat the spread of HIV/ AIDS must be re-evaluated with consideration for ways in which culture shapes risk perception. Societal factors, including practices and beliefs about sex, relationships, and condom-use lead to the inclusion, neglect, or exclusion of people, thereby shaping individual behavior in ways that are beyond individual control. ${ }^{27}$ Addressing social norms and values that negatively impact vulnerable populations such as women and children can effect change more rapidly than measures aimed at individual behavior. Thus, the ultimate aim should be to enable people to exert control over their own risk and to create an environment in which safer behavior can be practiced.

The role of public health policies and programs cannot and should not be limited to individual health behaviors. Despite the knowledge and experience in the field of public health with regards to the social determinants of disease, including food and nutrition, shelter, and employment, there has been little impact on HIV/AIDS policy and programming. ${ }^{28}$ In addition to recognizing the role of social context, policy and program implementation must also not occur in isolation. Inter-sectoral coordination is necessary to influence risk perception on a macro-level. Recognition of HIV/AIDS as not only a health problem, but also a social, economic, and development issue facilitates collaboration between different levels of government and civil society. However, it is important to stress that the application of public health efforts will differ from one region to another due to differences in demographics, political context, education levels, social service provision, geographic location, cultural beliefs and epidemic patterns, among other factors. For example, data suggests that in most cultures poverty exacerbates the spread of HIV, but there are also emerging epidemics among financially secure sectors of society partly because of the economic power to engage in risky behaviors such as buying sex or drugs. ${ }^{27}$ Whether safer behavior is more likely to occur as economic status increases depends on other factors such as social values, education and gender. This example illustrates the complexity of vulnerability, and the need to design interventions and policies that take regional variations into consideration.

Although targeting interventions to the changing needs of the communities for whom they are designed is crucial to program effectiveness, there are two vulnerable groups, in particular, that demand attention in all contexts: youth and females. Young people, both male and female, account for most of the current HIV/AIDS infections in the world today. ${ }^{27}$ The transition from childhood to adulthood can be fraught with difficulties such as lack of information, a desire to experiment, or feelings of "invulnerability". In most societies, young people have limited rights and are expected to be obedient to authority. ${ }^{27}$ This can impact their propensity to take part in risky behaviors and can diminish the potentially positive effects of health and social services. Youth are not only victims of the HIV/AIDS epidemic directly, but also indirectly. For example, it is estimated that the number of children and youth orphaned by HIV/AIDS will continue to rise for the next several years reaching 40 million by 2010 in African alone. ${ }^{29}$

Similarly, women and girls often face inequalities in access to education, in income and employment, and before the law, which places them at a disadvantage and reduces their ability to adopt safe behaviors. ${ }^{27}$ Reversing these inequalities will require cultural, legal, and policy-level changes. In the absence of policies and programs that bridge the age and gender gaps, efforts aimed at reducing the spread of HIV/AIDS may be ineffective and short-lived.

Keeping in mind the need to balance context specificity with consideration for cross-cutting issues like ageism and sexism, a multi-dimensional approach to HIV/AIDS mitigation is recommended. Such a model involves two dimensions: (1) persuading vulnerable population groups to change behavior, and (2) enabling safe behavior by changing societal and contextual factors that contribute to HIV/ AIDS transmission. ${ }^{25}$ Thus far, public health measures have focused on persuasion. This narrow focus on the individual has a limited impact on communities plagued by poverty, inequality, and injustice. Public health policies and programs must move to a paradigm of enablement and empowerment by addressing root causes. This is more challenging than behavior change programs because it requires collaboration across sectors and because the impact will not be evident in the near future. Cultural changes are gradual. Thus, the importance of long-term sustainable policies and programs cannot be overemphasized. There is a clear and definite need for global cooperation on this, especially considering that the lower economic status of developing countries will result in more acute challenges to the development and maintenance of necessary programming.

Within the discourse of public health, health risks have been individualized such that it is an individual's choice to engage in certain behaviors that cause the individual to acquire HIV/AIDS. ${ }^{5}$ This view has led to the labeling of particular groups of individuals and populations as "at-risk". 
However, the term "at-risk" is misleading considering that HIV/AIDS is now transmitted in a number of different ways, such as heterosexually and from mother to child. ${ }^{11}$ Awareness of risk is no longer sufficient. There must be acceptance at a cultural and social level that the risk of acquiring HIV/AIDS is not limited to particular individuals. HIV/AIDS is a threat to everyone and must be considered a shared responsibility. By reshaping risk perception, public health measures can effect change at the social level, where it matters.

Community-led approaches to HIV/AIDS mitigation have grown in popularity and are often quite innovative in their response. The advantages of such programs are that local knowledge is utilized and the focus is on communities as opposed to individuals. ${ }^{26}$ Community mobilization, paired with public health efforts can allow for a naturally evolving response to diversity and changing needs. Despite the great, untapped capacity of communities, local programs often continue to focus on behavior change out of context. However, proactive action rather than reactive action is required to ensure policy gets ahead of the epidemic.

\section{Application of the PAR model}

The spread of HIV/AIDS can be modeled much like an environmental disaster that is characterized by a slow onset and is exacerbated by human action or inaction. If humans can act as agents to mitigate the effects of hazards and disasters, accountability and responsibility must be acknowledged as not being limited to a selected group, but shared by all individuals and communities. ${ }^{22}$ Discourses surrounding the global HIV/ AIDS crisis must move in this direction; developing a recognition that HIV/AIDS is a shared responsibility requiring a coordinated response from all nations.

Considering the similarities between HIV/AIDS and other natural and environmental disasters, a disaster management approach to HIV/AIDS is needed to improve global responses. A disaster management framework can act as a policy and administrative tool to assist decision makers in determining the appropriate response to HIV/AIDS. For example, depending on the current level of HIV/AIDS impact and the potential for future disruption based on vulnerability, societies can be classified into one of three phases: (1) pre-disaster phase, (2) early-warning phase, or (3) disaster phase. In order to effectively utilize the PAR model within such a framework, there must be reliable and valid means for ongoing data collection in the nation, region, or community being studied. Data on social, economic, and health indicators paired with an understanding of the social and political context is necessary. In its simplest application, the PAR model can be used as a checklist in which existing factors are checked off, while factors that are not currently occurring in the target area are left blank. Used in this manner, the PAR model can indicate levels of vulnerability, or in other words the model will reveal which disaster phase the region is currently in. The model can also highlight which "pressures" should be released through addressing root causes that achieve safer conditions, thereby reducing the risk of disaster.

Nations with a low HIV/AIDS prevalence and low vulnerability for progression can be classified under Phase 1, the pre-disaster phase. With regards to environmental disasters, the pre-disaster phase is characterized by coordinated, focused development that reduces the likelihood of disastrous events through a collaborative response. In the context of HIV/AIDS, the pre-disaster phase involves risk reduction by focusing on high-risk and/or vulnerable populations, including not only sex workers and injection drug users, but also women and children. While environmental disasters are mitigated through activities like relocation or elevation of structures, construction of safe rooms, or vegetation management, HIV/ AIDS can be mitigated through policies and laws that promote equality, economic growth, and cultural norms associated with safe behaviors. In both cases, known hazards are reduced or eliminated as a prevention mechanism against disaster.

Nations with low HIV/AIDS prevalence and high vulnerability can be classified under Phase 2, the early-warning phase. Generally, the early-warning phase involves the implementation of existing response plans, relief assistance, and assessment of initial damages. Similarly, for HIV/AIDS mitigation the early-warning phase refers to a response involving heightened planning and expanded prevention activities that are focused on both high-risk groups and the general population.

Finally, nations with very high HIV/AIDS prevalence and high vulnerability can be classified as disaster situations (Phase 3). For environmental and natural disasters in Phase 3, there is a need to engage in search and rescue operations, begin reconstruction and rehabilitation, and conduct thorough assessments of damages. Similarly, recommendations for HIV/AIDS disasters include the mobilization of resources, reconstruction, and rehabilitation that involves ongoing treatment, care, and prevention efforts. Unlike the initial two phases of the disaster management framework, the third phase is not focused on prevention, but rather on treatment and alleviation.

\section{Conclusion}

The HIV/AIDS pandemic can be modeled much like an environmental or natural disaster that causes social disruption 
and loss of life. Despite the harrowing impact of HIV/AIDS globally, the response from governments has been slow and ineffective, characterized by cultural norms focused on choice, blame, and individual responsibility.

A disaster management approach to prevention, treatment, and mitigation can ensure that HIV/AIDS becomes a priority and is addressed with the same concern and attention as other environmental disasters. Utilization of the PAR model can direct and enhance disaster management efforts. By using the PAR model as a guide, public health departments can work towards addressing one or more of the three features of increasing vulnerability, namely: (1) root causes such as poverty, access to resources, and gender inequality; (2) dynamic pressures such as migration and employment; and (3) unsafe conditions such as sanitation, access to healthcare, and stigma.

In addition to reducing vulnerability by addressing root causes, particular attention can be paid to developing methods for changing perceptions of risk. By impacting cultural and social expectations, individuals will be able to more readily adopt safer sex behaviors. The development of policies and programs addressing the issues in context, as opposed to individual behaviors alone, allows for effective public health intervention - which is needed if we are to prevent the HIV/AIDS pandemic from becoming a full blown catastrophic disaster.

\section{Disclosure}

The authors report no conflicts of interest in this work.

\section{References}

1. UNAIDS. AIDS epidemic update. Geneva, Switzerland: UNAIDS, c2007 [cited 2008 Sep 10]. Available from: http://data.unaids.org/pub/ EPISlides/2007/2007_epiupdate_en.pdf.

2. Wildavsky A, Dake K. Theories of risk perception: Who fears what and why? Daedalus. 1990;119:41-59.

3. Elliott A. Beck's sociology of risk: A critical assessment. Sociology. 2002;36:293-315.

4. Douglas M, Wildavsky A. Risk and culture - An essay on the selection of technical and environmental dangers. Berkeley, CA: University of California Press, Ltd; 1982.

5. Harthorn B, Oaks L. Risk, culture, and health inequality: Shifting perceptions of danger and blame. Connecticut: Praeger Publishers; 2003.

6. Lupton D. Risk. London: Routledge; 1999.

7. Bloor M. The sociology of HIV transmission. London: SAGE Publications Ltd; 1995.

8. Barnett T, Whiteside A. AIDS in the twenty-first century. New York, NY: Palgrave Macmillan; 2002.
9. Petchesky R. Global prescriptions: Gendering health and human rights. London, UK: Zed Books; 2003.

10. World Health Organization. HIV/AIDS epidemiological surveillance report for the WHO African region. Geneva, Switzerland: WHO; 2005. [cited Sep 10]. Available from: http://www.who.int/hiv/pub/ epidemiology/hivinafrica2005e_web.pdf.

11. Beck E, Mays N, Whiteside A, et al. The HIV pandemic: Local and global implications. New York, NY: Oxford University Press; 2006.

12. Schoepf B. Uganda: Lessons for AIDS control in Africa. Review of African Political Economy; 2003:30:553-72.

13. Shaluf I, Ahmadun F, Said A. Review of disaster and crisis. Disaster Prevention and Management; 2003:12:24-32.

14. Wisner B, Blaikie P, Cannon T, et al. At risk: Natural disasters, people's vulnerability and disasters. Second Ed. London, UK: Routledge; 2004.

15. Cannon, T. Vulnerability analysis and the explanation of 'natural' disaster. In: Varley A (ed). Disasters, Development and Environment. Chichester: John Wiley and Sons Ltd; 1994:p.13-30.

16. Farmer P. Woman, poverty, and AIDS (Chapter 1). In: Farmer P, Conors M, Simmons J (eds). Women, poverty, and AIDS - Sex, drugs and structural violence. Maine: Common Courage Press; 1996:p. 3-38.

17. UNAIDS. Overview of the global AIDS epidemic. Report on the global AIDS epidemic. Geneva, Switzerland: UNAIDS, c2006 [cited 2008 Sep 10]. Available from: http://www.unaids.org/en/HIV_data/ 2006GlobalReport/default.asp.

18. Barnett A, Blaikie P. AIDS as a long wave disaster. In: Varley A (ed). Disasters, Development and Environment. Chichester: John Wiley and Sons Ltd; 1994:p. 139-62.

19. Elioutina S, Tarasov V. Current state and perspectives of healthcare informatics in Russia. Int J Biomed Comput. 1995;39:163-7.

20. Inciardi J, Surrat H, Telles P. Sex, drugs, and HIV/AIDS in Brazil. New York, NY. Westview Press; 2000.

21. Smallman S. The AIDS pandemic in Latin America. Chapel Hill, NC: The University of North Carolina Press; 2007.

22. Stefoff R. Environmental disasters. New York, NY: Chelsea House Publications; 1994

23. World Health Organization. Disaster preparedness and response. WHO, c2007 [cited 2008 Mar 13]. Available from: http://www.euro.who. int/emergencies.

24. Black J, Bryant J, Thompson S. Introduction to media communication. New York. NY: McGraw Hill; 1998.

25. Chatterjee, N. AIDS-related information exposure in the mass media and discussion within social networks among married women in Bombay, India. AIDS Care. 1999;4:443-6.

26. Parker R. The social and cultural construction of sexual risk, or how to have (sex) research in an epidemic. In: ten Brummelhuis $\mathrm{H}$, Herdt G (eds). Culture and Sexual Risk: Anthropological Perspectives on AIDS, Amsterdam. Holland: Gordon and Breach Publishers; 1995 p. 257-69.

27. UNAIDS. Expanding the global response to HIV/AIDS through focused action: Reducing risk and vulnerability: definitions, rationale, and pathways. UNAIDS, c1998 [cited 2008 Sep 10]. Available from: http://www.unaids.org/en/.

28. Gillespie S, AIDS. AIDS, poverty, and hunger: Challenges and responses. Highlights of the 2006 International Conference on HIV/ AIDS and Food and Nutrition Security, Durban, South Africa, April 14-6, 2005.

29. Foster G, Williamson J. A review of current literature on the impact of HIV/AIDS on children in Sub-Sahara Africa. AIDS. 2000;14(Suppl 3):S275-84. 